\title{
Nanopyramid With High Purity And Their Local Plasmonic Properties
}

\author{
Man Qiu ${ }^{1}$ and $\mathrm{Li} \mathrm{Yu}{ }^{1}$ \\ ${ }^{1}$ Beijing University of Posts and Telecommunications
}

March 19, 2021

\begin{abstract}
$\mathrm{Au}$ nanopyramid particles (Au NBPs) are highly desirable for its remarkable optical properties such as long-range tunable resonance. It has wide applications in room-temperature bioimaging probes and bioanalytical sensors. In this paper, we synthesize Au NBPs with a purity of $95 \%$, and obtain the optical response of Au NBP in near infrared regime. We find that Au NBPs have small mode volume of electric field which can lead to the strong coupling with quantum dots at room temperature. It provides novel applications for Au NBPs in fields of materials, biomedical science, and quantum information
\end{abstract}

\section{Hosted file}

$\backslash$ begin\{CJK\}\{UTF8\}\{gbsn\}\end\{CJK\}\selectlanguage\{english\}_EDITED.docx available at https: } //authorea.com/users/399551/articles/514401-nanopyramid-with-high-purity-and-theirlocal-plasmonic-properties 